\title{
Gallbladder Perforation, CTCAE 5.0
}

National Cancer Institute

\section{Source}

National Cancer Institute. Gallbladder Perforation, CT CAE 5.0. NCI Thesaurus. Code C146708.

A disorder characterized by a rupture in the gallbladder wall. 